\title{
Maghemite/Polyvinylidene Fluoride Nanocomposite for Transparent, Flexible Triboelectric Nanogenerator and Noncontact Magneto-triboelectric Nanogenerator
}

Bushara Fatma ${ }^{\dagger *}$, Ritamay Bhunia ${ }^{\dagger}$, Shashikant Gupta ${ }^{\dagger}$, Amit Verma $^{\ddagger}$, Vivek Verma ${ }^{\dagger}$, Ashish Garg ${ }^{\dagger *}$

${ }^{\dagger}$ Department of Material Science and Engineering, Faculty Building, Indian Institute of Technology Kanpur, Kalyanpur, Kanpur 208016, India.

Department of Electrical Engineering, ACES Building, Indian Institute of Technology Kanpur, Kalyanpur, Kanpur 208016, India.

*E-mail: bushara@iitk.ac.in

(a)

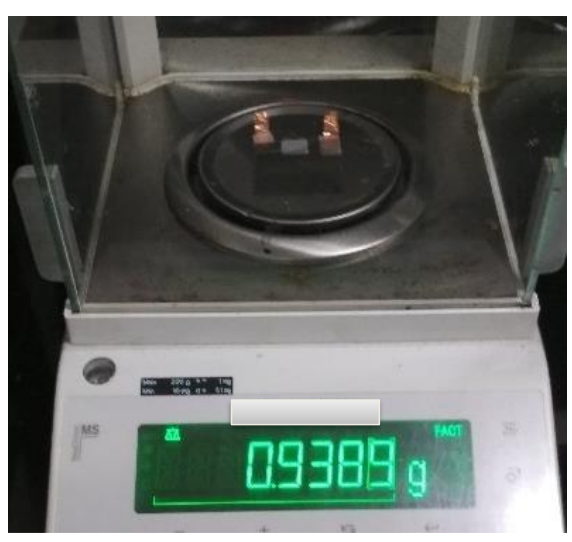

(b)

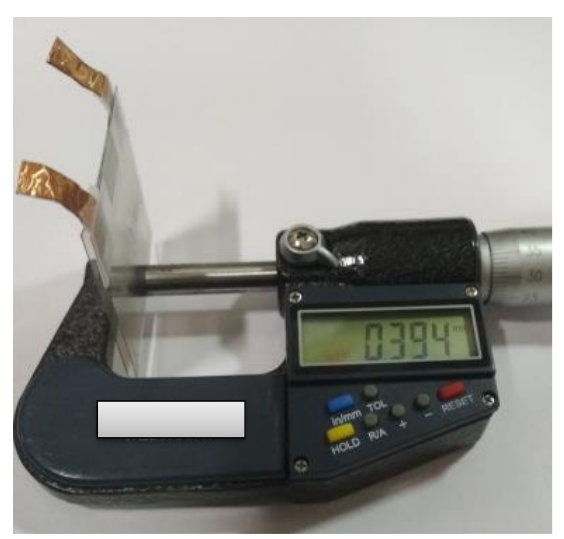

Figure S1. (a) Weight and (b) thickness of complete TENG device is $938 \mathrm{mg}$ and $394 \mu \mathrm{m}$, respectively.
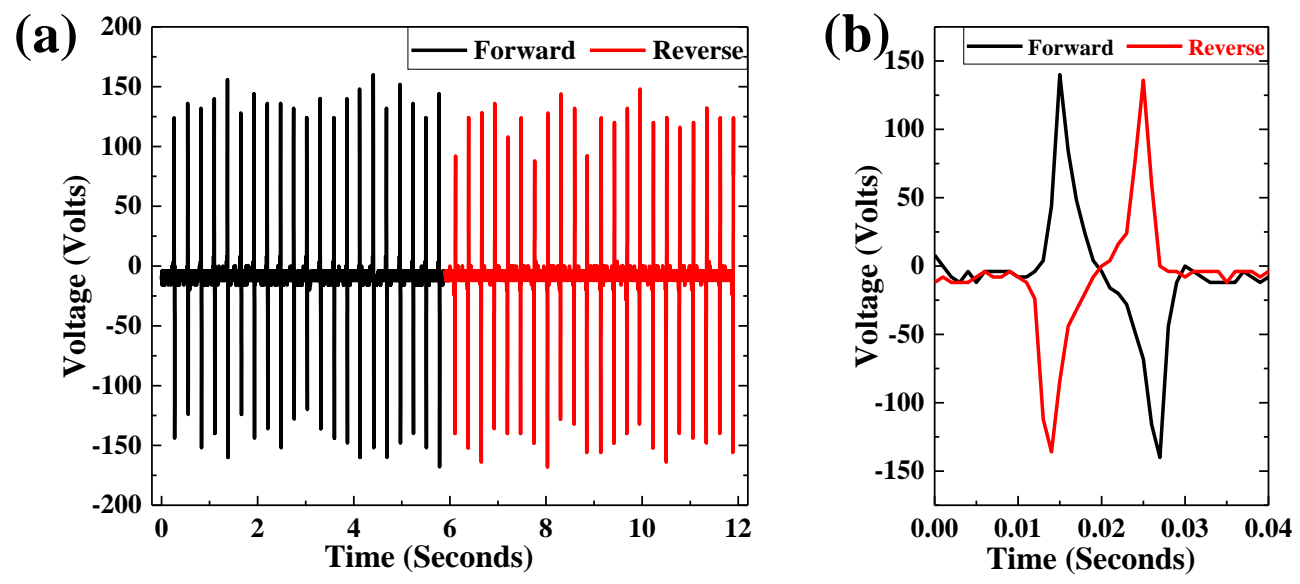

Figure S2. (a) Switching polarity test of TENG made of pure PVDF. (b) Zoomed image of single peak (right) under forward and reverse connection. 


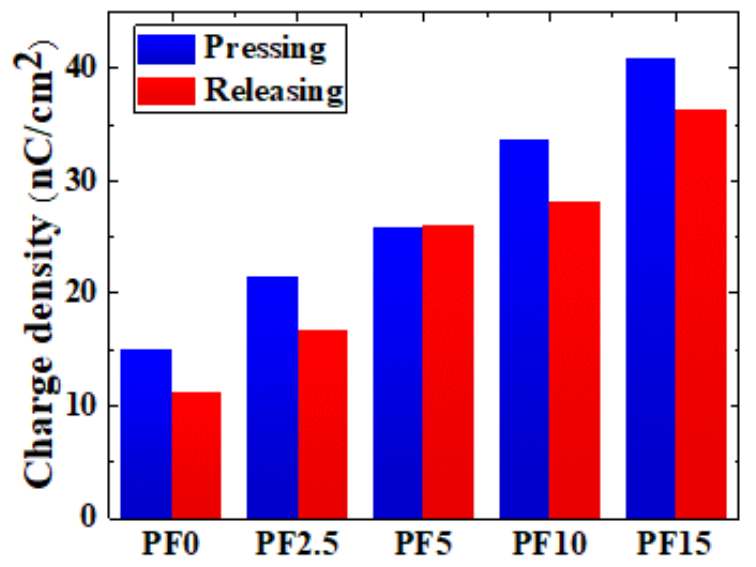

Figure S3. Charge density of composites with different concentrations (0, 2.5, 5, 10 and $15 w t \%)$ of nanoparticles.

(a)

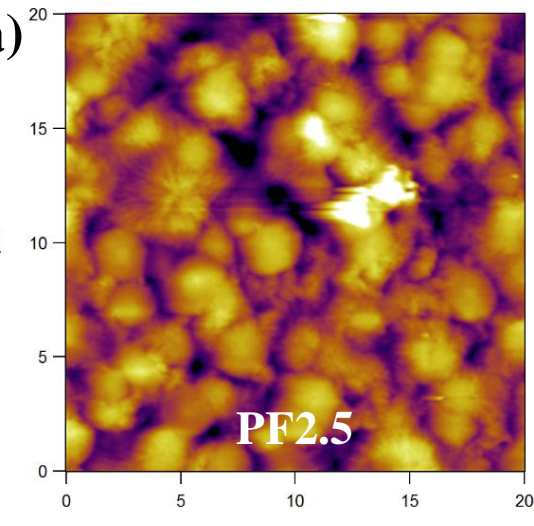

(c)

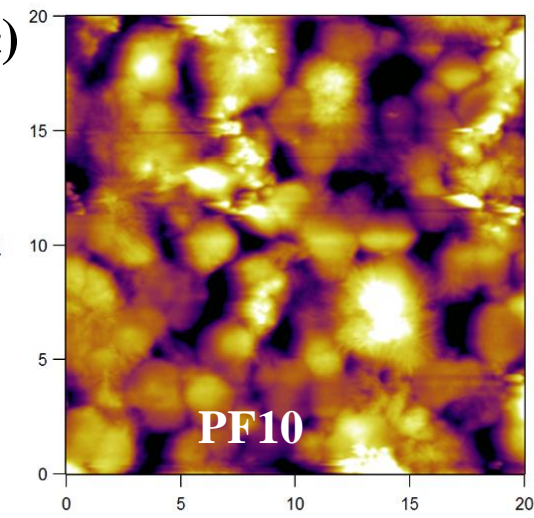

(e)
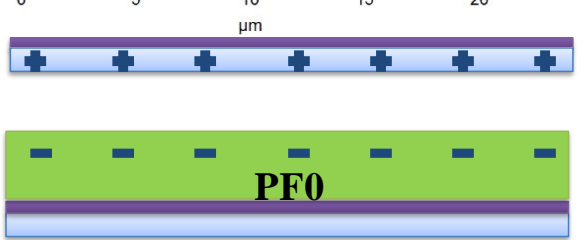

(b)
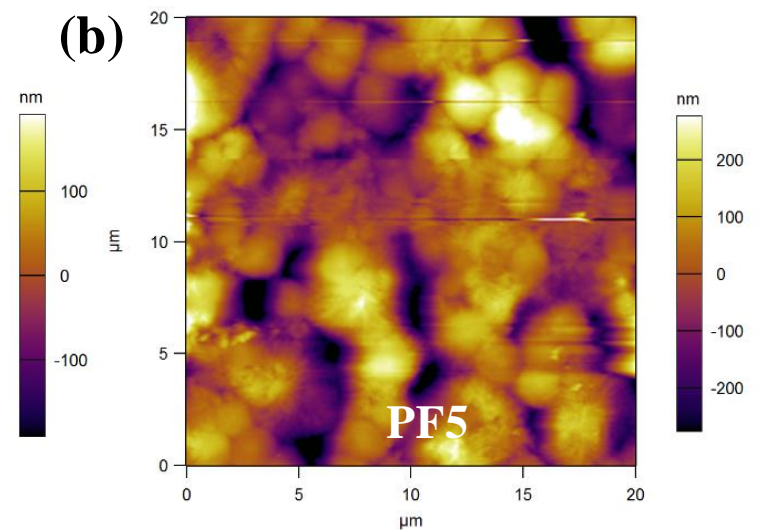

(d)
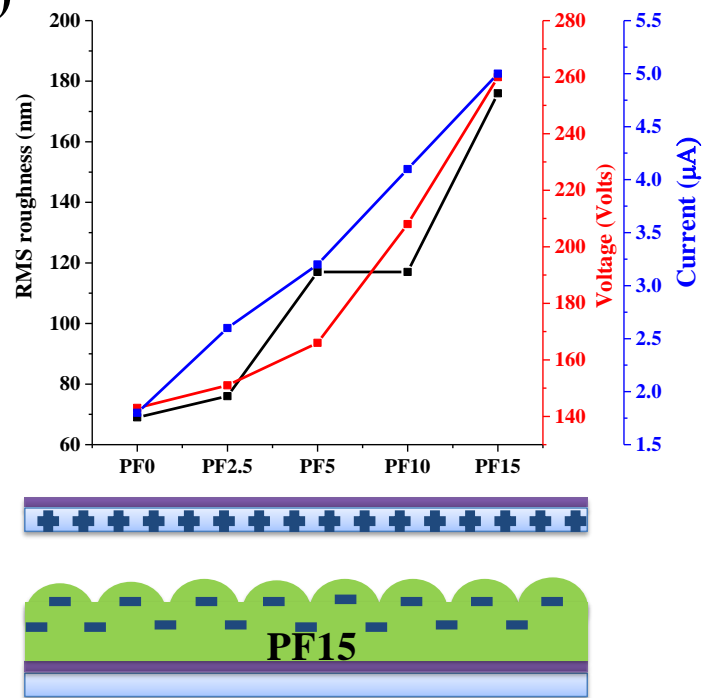

Figure S4. AFM image $\left(20 \times 20 \mu \mathrm{m}^{2}\right)$ showing surface roughness of (a) PF2.5, (b) PF5 and (c) PF10 composites. (d) Surface roughness variation with addition of $\gamma-\mathrm{Fe}_{2} \mathrm{O}_{3}$ to $\mathrm{PVDF}$ from $0 \mathrm{wt} \%$ to $15 \mathrm{wt} \%$. (e) Schematic representation of increase in surface charge with an increase in surface roughness. 
(a)

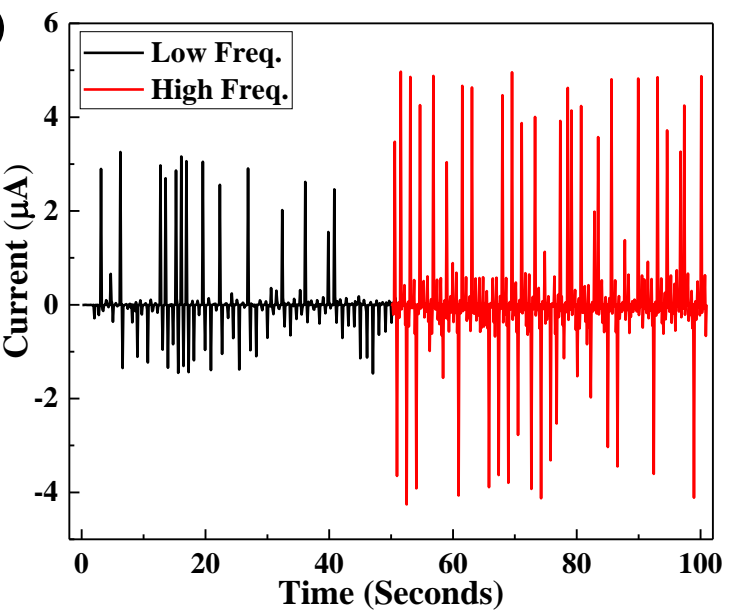

(b)

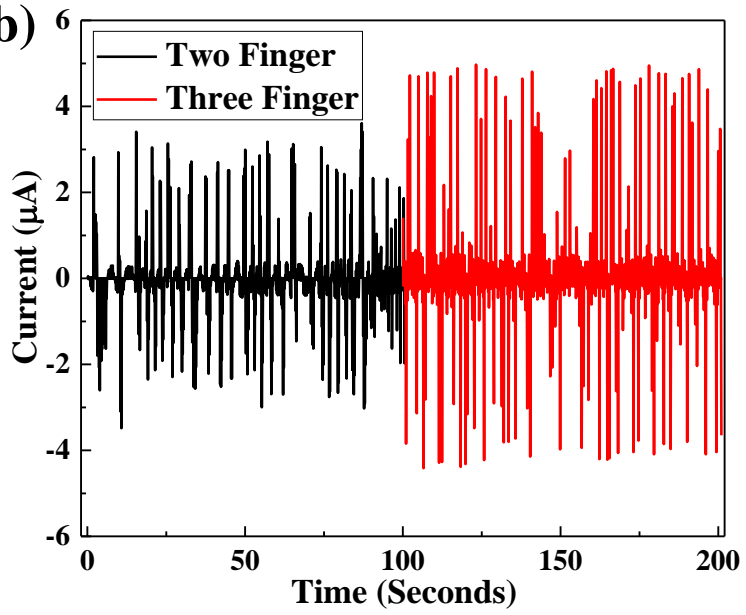

(c)

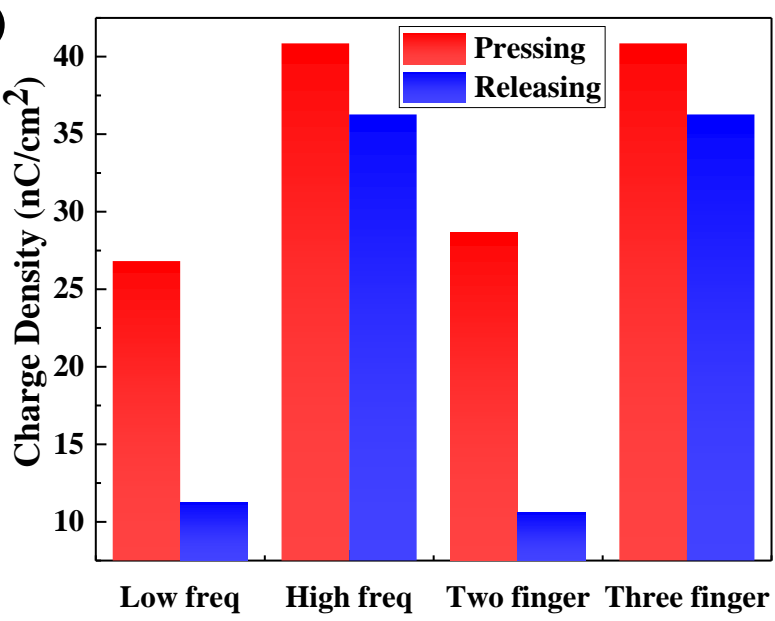

Figure S5. Output current for (a) low frequency and high frequency tapping and (b) two finger and three finger tapping. (c) Charge density during pressing and releasing for low frequency and high frequency tapping as well as two finger and three finger tapping.

(a)

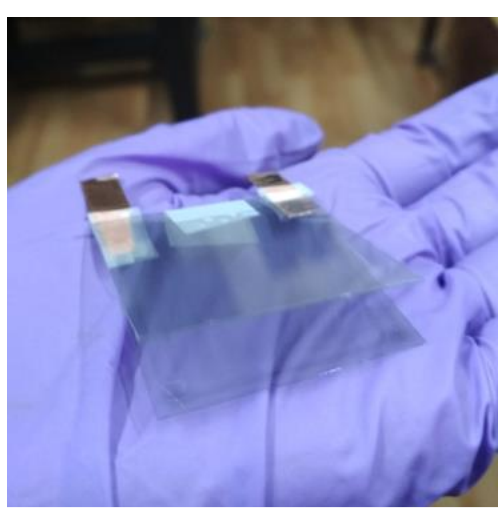

(b)

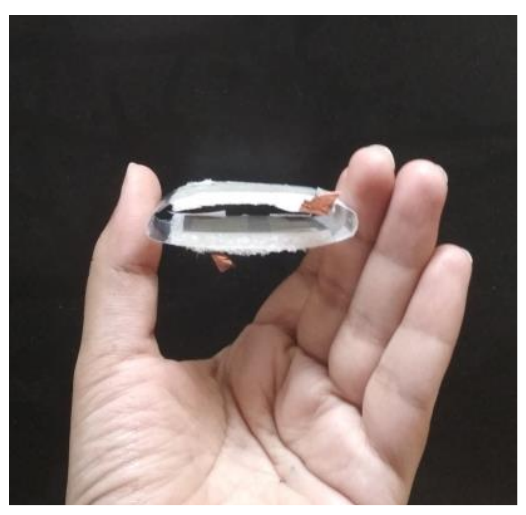

Figure S6. TENG device structure (a) when integrated to the rotary pump for scavenging energy from its high frequency vibrations and (b) when integrated to sole of footwear as wearable electronics for harvesting walking and running motions. 
Table S1. Comparison of our device output with other devices reported in literature.

\begin{tabular}{|c|c|c|c|c|c|c|c|c|c|}
\hline \multirow{2}{*}{$\begin{array}{l}\text { S. } \\
\text { No }\end{array}$} & \multirow{2}{*}{$\begin{array}{c}\text { Triboelectric } \\
\text { Material }\end{array}$} & \multirow{2}{*}{$\begin{array}{l}\text { Electrode } \\
\text { Material }\end{array}$} & \multirow{2}{*}{$\begin{array}{l}\text { Mode of } \\
\text { Operation }\end{array}$} & \multirow{2}{*}{$\begin{array}{l}\text { Output } \\
\text { Voltage } \\
\text { (Volts) }\end{array}$} & \multirow{2}{*}{$\begin{array}{c}\text { Output } \\
\text { Current } \\
(\boldsymbol{\mu A})\end{array}$} & \multicolumn{3}{|c|}{ Application } & \multirow[t]{2}{*}{ Ref. } \\
\hline & & & & & & Type & $\begin{array}{l}\text { Output } \\
\text { Voltage } \\
\text { (Volts) } \\
\end{array}$ & $\begin{array}{c}\text { Output } \\
\text { Current } \\
(\mu \mathrm{A})\end{array}$ & \\
\hline 1. & $\begin{array}{c}\text { Human Hair Film, } \\
\text { Kapton } \\
\text { (Polyimide) }\end{array}$ & ITO & $\begin{array}{l}\text { Contact- } \\
\text { separation }\end{array}$ & $\sim 55$ & $\sim 5.5$ & --- & --- & -- & 1 \\
\hline 2. & $\begin{array}{l}\text { Nylon+Soy } \\
\text { protein/lignin, } \\
\text { Polyimide }\end{array}$ & $\mathrm{Cu}$ & $\begin{array}{l}\text { Contact- } \\
\text { separation }\end{array}$ & $\sim 5$ & $\sim 0.6$ & $\begin{array}{l}\text { Biomechanical } \\
\text { energy harvesting }\end{array}$ & $\sim 1.2$ & --- & 2 \\
\hline 3. & $\mathrm{PVDF}+\mathrm{Fe}_{3} \mathrm{O}_{4}, \mathrm{Al}$ & $\mathrm{Al}$ & $\begin{array}{l}\text { Contact- } \\
\text { separation }\end{array}$ & $\sim 138$ & $\sim 6$ & --- & --- & --- & 3 \\
\hline 4. & $\begin{array}{l}\text { PTFE/PET, CNT } \\
\text { on stainless steel }\end{array}$ & $\begin{array}{l}\mathrm{Au}, \mathrm{CNT} \text { on } \\
\text { stainless steel }\end{array}$ & $\begin{array}{l}\text { Contact- } \\
\text { separation }\end{array}$ & $\sim 3$ & $\sim 0.16$ & --- & --- & --- & 4 \\
\hline 5. & $\begin{array}{l}\text { Egg white, chitin, } \\
\text { cellulose, raw silk, } \\
\text { rice paper }\end{array}$ & $\mathrm{Mg}$ & $\begin{array}{l}\text { Contact- } \\
\text { separation }\end{array}$ & $\sim 50$ & $\sim 0.5$ & $\begin{array}{l}\text { Biodegradable } \\
\text { Implants }\end{array}$ & $\sim 5$ & --- & 5 \\
\hline 6. & Silk, PET & ITO & $\begin{array}{l}\text { Contact- } \\
\text { separation }\end{array}$ & $\sim 268$ & $\sim 5.8$ & $\begin{array}{l}\text { Optical display, } \\
\text { micromechanical } \\
\text { device }\end{array}$ & --- & --- & 6 \\
\hline 7. & $\begin{array}{c}\text { PVDF, PDMS, } \\
\text { ZnO }\end{array}$ & $\mathrm{C}, \mathrm{Al}$ & $\begin{array}{l}\text { Contact- } \\
\text { separation }\end{array}$ & $\sim 35.7$ & --- & --- & --- & --- & 7 \\
\hline 8. & $\begin{array}{c}\text { Cellulose, } \\
\text { Cellulose+Ag }\end{array}$ & $\begin{array}{c}\text { Cellulose+ Ag } \\
\text { nanowire }\end{array}$ & $\begin{array}{l}\text { Contact- } \\
\text { separation }\end{array}$ & $\sim 21$ & $\sim 2.5$ & Writable surface & --- & $\sim 30 \mathrm{nA}$ & 8 \\
\hline 9. & $\begin{array}{c}\text { PDMS and } \\
\text { PDMS/MWCNT }\end{array}$ & $\begin{array}{l}\text { Metal and } \\
\text { PDMS/MW- } \\
\text { CNT }\end{array}$ & $\begin{array}{l}\text { Contact- } \\
\text { separation }\end{array}$ & $\sim 30$ & $\sim 2.5$ & --- & --- & --- & 9 \\
\hline 10. & Starch paper & Metal wire & $\begin{array}{l}\text { Single } \\
\text { electrode }\end{array}$ & $\sim 14$ & --- & $\begin{array}{l}\text { Biomechanical } \\
\text { harvesting }\end{array}$ & $\sim 7.5$ & --- & 10 \\
\hline 11. & PDMS, Silk & ITO & $\begin{array}{l}\text { Single } \\
\text { electrode }\end{array}$ & $\sim 65$ & --- & $\begin{array}{l}\text { Wind Energy } \\
\text { harvesting }\end{array}$ & $\sim 65$ & --- & 11 \\
\hline 12. & $\begin{array}{c}\text { Super- } \\
\text { hydrophobic } \\
\text { coating on PET }\end{array}$ & $\mathrm{Au}$ & $\begin{array}{l}\text { Single } \\
\text { electrode }\end{array}$ & $\sim 22$ & $\sim 8$ & $\begin{array}{l}\text { Water energy } \\
\text { harvesting }\end{array}$ & $\sim 15$ & $\sim 4$ & 12 \\
\hline 13. & PVA/PEI, PET & ITO, Ag & $\begin{array}{l}\text { Contact- } \\
\text { separation }\end{array}$ & $\sim 80$ & $\sim 5$ & $\begin{array}{l}\text { Biomechanical } \\
\text { harvesting }\end{array}$ & $\sim 30$ & $\sim 3.2$ & 13 \\
\hline 14. & Paper, PVC, PET & $\begin{array}{l}\text { Paper+ Ag } \\
\text { nanowire }\end{array}$ & $\begin{array}{c}\text { Free standing } \\
\text { triboelectric } \\
\text { layer }\end{array}$ & $\sim 100$ & --- & Soft tissue paper & --- & --- & 14 \\
\hline 15. & $\begin{array}{l}\text { Fluoropolymer, } \\
\text { PET }\end{array}$ & $\mathrm{Ag}$ & $\begin{array}{l}\text { Contact- } \\
\text { separation }\end{array}$ & $\sim 50$ & $\sim 5.5$ & $\begin{array}{l}\text { Water energy } \\
\text { harvesting }\end{array}$ & $\sim 4$ & --- & 15 \\
\hline 16. & Silk+PET, PET & ITO & $\begin{array}{l}\text { Contact- } \\
\text { separation }\end{array}$ & $\sim 210$ & --- & --- & --- & --- & 16 \\
\hline 17 & $\begin{array}{l}\mathrm{ZnO+PVDF}, \\
\text { PTFE }\end{array}$ & $\mathrm{Au}$ & $\begin{array}{l}\text { Contact- } \\
\text { separation }\end{array}$ & $\sim 75$ & $\sim 0.7$ & --- & --- & --- & 17 \\
\hline 18 & PVDF, Polyimide & $\begin{array}{l}\mathrm{MoS}_{2}+ \\
\text { Cellulose }\end{array}$ & $\begin{array}{l}\text { Contact- } \\
\text { separation }\end{array}$ & $\sim 50$ & $\sim 30 \mathrm{nA}$ & Writable Surface & $\sim 0.5$ & --- & 18 \\
\hline 19 & $\begin{array}{c}\text { Eu doped PVDF, } \\
\text { Al foil }\end{array}$ & $\begin{array}{c}\text { Al foil, } \\
\text { Stainless steel } \\
\text { plate }\end{array}$ & $\begin{array}{l}\text { Contact- } \\
\text { separation }\end{array}$ & $\sim 120$ & $\sim 8$ & --- & --- & --- & 19 \\
\hline 20 & $\begin{array}{c}\text { Polycaprolactone+ } \\
\text { GO, cellulose }\end{array}$ & $\mathrm{Au}$ & $\begin{array}{c}\text { Contact- } \\
\text { separation }\end{array}$ & $\sim 120$ & $\sim 4$ & --- & --- & --- & 20 \\
\hline
\end{tabular}




\begin{tabular}{|c|c|c|c|c|c|c|c|c|c|}
\hline 21. & $\begin{array}{c}\text { PVDF+ } \\
\text { Maghemite/ PET }\end{array}$ & ITO & $\begin{array}{c}\text { Contact- } \\
\text { separation }\end{array}$ & $\sim 250$ & $\sim 5$ & $\begin{array}{l}\text { Harvesting high } \\
\text { frequency } \\
\text { vibrations }\end{array}$ & $\sim \mathbf{5 0}$ & $\sim 4.7$ & $\begin{array}{l}\text { This } \\
\text { work }\end{array}$ \\
\hline
\end{tabular}

\section{References:}

(1) Jayaweera, E. N.; Wijewardhana, K. R.; Ekanayaka, T. K.; Shahzad, A.; Song, J.-K., Triboelectric Nanogenerator Based on Human Hair. ACS Sustainable Chem. Eng. 2018, 6 (5), 63216327, DOI 10.1021/acssuschemeng.8b00136.

(2) An, S.; Sankaran, A.; Yarin, A. L., Natural Biopolymer-Based Triboelectric Nanogenerators via Fast, Facile, Scalable Solution Blowing. ACS Appl. Mater. Interfaces 2018, 10 (43), 37749-37759, DOI 10.1021/acsami.8b15597.

(3) Im, J.-S.; Park, I.-K., Mechanically Robust Magnetic $\mathrm{Fe}_{3} \mathrm{O}_{4} \mathrm{Nan}_{\text {naparticle/Polyvinylidene }}$ Fluoride Composite Nanofiber and Its Application in a Triboelectric Nanogenerator. ACS Appl. Mater. Interfaces 2018, 10 (30), 25660-25665, DOI 10.1021/acsami.8b07621.

(4) Oguntoye, M.; Johnson, M.; Pratt, L.; Pesika, N. S., Triboelectricity Generation from Vertically Aligned Carbon Nanotube Arrays. ACS Appl. Mater. Interfaces 2016, 8 (41), 27454-27457, DOI 10.1021/acsami.6b11457.

(5) Jiang, W.; Li, H.; Liu, Z.; Li, Z.; Tian, J.; Shi, B.; Zou, Y.; Ouyang, H.; Zhao, C.; Zhao, L.; Sun, R.; Zheng, H.; Fan, Y.; Wang, Z. L.; Li, Z., Fully Bioabsorbable Natural-Materials-Based Triboelectric Nanogenerators. Adv Mater 2018, 30 (32), e1801895, DOI 10.1002/adma.201801895.

(6) Zhang, X.-S.; Brugger, J.; Kim, B., A silk-fibroin-based transparent triboelectric generator suitable for autonomous sensor network. Nano Energy 2016, 20, 37-47, DOI

10.1016/j.nanoen.2015.11.036.

(7) Yang, X.; Daoud, W. A., Triboelectric and Piezoelectric Effects in a Combined TriboPiezoelectric Nanogenerator Based on an Interfacial ZnO Nanostructure. 2016, 26 (45), 8194-8201, DOI 10.1002/adfm.201602529.

(8) Kim, I.; Jeon, H.; Kim, D.; You, J.; Kim, D., All-in-one cellulose based triboelectric nanogenerator for electronic paper using simple filtration process. Nano Energy 2018, 53, 975-981, DOI 10.1016/j.nanoen.2018.09.060.

(9) Zhu, Y.; Yang, B.; Liu, J.; Wang, X.; Wang, L.; Chen, X.; Yang, C., A flexible and biocompatible triboelectric nanogenerator with tunable internal resistance for powering wearable devices. Sci Rep 2016, 6, 22233, DOI 10.1038/srep22233.

(10) Zhu, Z.; Xia, K.; Xu, Z.; Lou, H.; Zhang, H. J. N. R. L., Starch Paper-Based Triboelectric Nanogenerator for Human Perspiration Sensing. 2018, 13 (1), 365, DOI 10.1186/s11671-018-2786-

9.

(11) Zhang, X.; Guo, Y.; Wang, Y.; Zhang, H.; Brugger, J. In A transparent silk-fibroin-based triboelectric microgenerator for airflow energy harvesting, 2017 IEEE 12th International Conference on

Nano/Micro Engineered and Molecular Systems (NEMS), 9-12 April 2017; 2017; pp 65-68.

(12) Xiong, J.; Lin, M.-F.; Wang, J.; Gaw, S. L.; Parida, K.; Lee, P. S., Wearable All-Fabric-Based

Triboelectric Generator for Water Energy Harvesting. 2017, 7 (21), 1701243, DOI 10.1002/aenm.201701243. 
(13) Wang, L.; Daoud, W. A., Highly Flexible and Transparent Polyionic-Skin Triboelectric Nanogenerator for Biomechanical Motion Harvesting. 2019, 9 (5), 1803183, DOI 10.1002/aenm.201803183.

(14) Wu, C.; Kima, T. W.; Sung, S.; Park, J. H.; Li, F., Ultrasoft and cuttable paper-based triboelectric nanogenerators for mechanical energy harvesting. Nano Energy 2018, 44, 279-287, DOI 10.1016/j.nanoen.2017.11.080.

(15) Ha, J.; Chung, J.; Kim, S.; Kim, J. H.; Shin, S.; Park, J. Y.; Lee, S.; Kim, J.-B., Transferprintable micropatterned fluoropolymer-based triboelectric nanogenerator. 2017, 36 (Energy 32 2007), 126-133, DOI 10.1016/j.nanoen.2017.04.009.

(16) Liu, C.; Li, J.; Che, L.; Chen, S.; Wang, Z.; Zhou, X., Toward large-scale fabrication of triboelectric nanogenerator (TENG) with silk-fibroin patches film via spray-coating process. Nano Energy 2017, 41, 359-366, DOI 10.1016/j.nanoen.2017.09.038.

(17) Singh, H. H.; Khare, N., Flexible ZnO-PVDF/PTFE based piezo-tribo hybrid nanogenerator. Nano Energy 2018, 51, 216-222, DOI 10.1016/j.nanoen.2018.06.055.

(18) Sahatiya, P.; Kannan, S.; Badhulika, S., Few layer MoS2 and in situ poled PVDF nanofibers on low cost paper substrate as high performance piezo-triboelectric hybrid nanogenerator: Energy harvesting from handwriting and human touch. Applied Materials Today 2018, 13, 91-99, DOI 10.1016/j.apmt.2018.08.009.

(19) Kim, H.-S.; Park, I.-K., Enhanced output power from triboelectric nanogenerators based on electrospun Eu-doped polyvinylidene fluoride nanofibers. Journal of Physics and Chemistry of Solids 2018, 117, 188-193, DOI 10.1016/j.jpcs.2018.02.045.

(20) Parandeh, S.; Kharaziha, M.; Karimzadeh, F., An eco-friendly triboelectric hybrid nanogenerators based on graphene oxide incorporated polycaprolactone fibers and cellulose paper. Nano Energy 2019, 59, 412-421, DOI 10.1016/j.nanoen.2019.02.058. 